\title{
Forschende Lehre in einer polarisierten Gesellschaft - Perspektiven aus einer angewandten kritischen Geographie
}

\author{
"valentin.domann@geo.hu-berlin.de, Geographisches Institut, Humboldt-Universität Berlin \\ ** stephan.liebscher@fu-berlin.de, Institut für Geographische Wissenschaften, Freie Universität Berlin
}

eingereicht am: 20.12.2019, akzeptiert am: 08.02.2020

\begin{abstract}
Der Artikel geht der Frage nach, wie geographische Lehre an der Hochschule in Zeiten eines allgegenwärtigen Rechtsrucks kritische Perspektiven anregen und zugleich in stadtgesellschaftlichen Polarisierungsprozessen emanzipatorisch intervenieren kann. Hierfür werden Erfahrungen aus zwei Lehrforschungsprojekten aus Chemnitz und Berlin dialogisch reflektiert. Dabei werden Potenziale von verschiedenen Ansätzen der geographischen Forschung und Lehre, die sich partizipatorischen und transformativen Method(ologi)en bedienen, wie Aktionsforschung und Urban Transition Lab, diskutiert.
\end{abstract}

Keywords: forschende Lehre, Urban Transition Lab, Aktionsforschung, Solidarität, Rechtspopulismus, Geographie

\section{Research-led teaching in a polarized society - perspectives of a practical-critical geography}

The article examines how academic teaching in geography can stimulate critical perspectives in times of an omnipresent political shift to the right and how it can intervene in processes of polarization of urban societies in emancipatory ways. For this purpose, experiences from two research-led seminars in Chemnitz and Berlin are reflected in dialogue. The article also discusses potentials of different approaches using participatory and transformative methodologies in geographical research and teaching, such as action research and urban transition labs.

Keywords: research-led teaching, Urban Transition Lab, action research, solidarity, right-wing populism, geography

1 Ausgangslage: Transformative Ansprüche der kritisch-geographischen Lehre und Chemnitzer Ausschreitungen als Stein des Anstoßes

Die kritische Geographie feiert beim Verfassen dieser Zeilen, ohne große Festakte, ihr fünfzigstes Jubiläum. Nimmt man die Gründungsmythen beim Wort, fiel 1969 der Startschuss für die Entwicklung einer eigenständigen Denktradition innerhalb der geographischen Wissenschaften, zunächst unter dem Label der radical geography. Einhergehend mit der Diversifizierung, Institutionalisierung und Professionalisierung der kritischen Geographie, stellt sich zuletzt vermehrt die Frage danach, wie sich das ursprüngliche Versprechen einer aktionsorientierten und solidarischen Wissenschaft aus dem Hörsaal einer Bologna-Universität noch einlösen lässt (vgl. Castree 2000).

In diesem Zusammenhang wird vermehrt die Rolle von kritischen Geographinnen und Geographen in der Hochschullehre diskutiert (vgl. etwa Catungal 2019). Zentrale Überlegungen werden hierzu der kritischen Pädagogik, etwa von Henry Giroux, entlehnt, die das Seminar als Raum konzipieren, der auf vielerlei Art und Weise mit der gesellschaftlichen Realität verbunden ist (vgl. Heyman 2001). Allein die Thematisierung gesellschaftlicher Missstände unter „klassischen Lehrbedingungen“, würde demnach nicht weit genug gehen, gar Gefahr laufen, Wissen zu objektifizieren und das Gegebene als statisch zu vermitteln: „no matter how terrible some of the problems we discuss in our classrooms may be, they are almost invariably situated as being someone else's problems, lived somewhere else" (Hay 2001: 169). Folgend gelte es, Formate (weiter-) zu entwickeln, die Studierenden eine aktive Rolle in der Wissensproduktion zuweisen (vgl. Heyman 2001).

Unter dieser Prämisse bieten insbesondere Methoden des forschenden Lernens vielversprechende $\mathrm{Zu}$ gänge zur Seminargestaltung, da Studierende hier im 
Rahmen der Lehrveranstaltung angehalten sind, alle Phasen einer empirischen Forschung selbst durchzuführen und der Seminarkontext der gemeinsamen Reflexion dient (vgl. Reinmann 2016). Weiterhin kann kritisch-geographische Lehre auf Ideen aufbauen, die, entgegen einem positivistischen Wissenschaftsverständnis, die transformativen und progressiven Momente von Forschung herausheben. Die hier vorgestellten Seminarkonzepte profitierten insbesondere von den mehrheitlich positiven Erfahrungen der Autoren mit Mitteln der Aktionsforschung und der Methodologie der Urban Transition Labs.

Die beiden Ansätze versammeln jeweils eine Reihe unterschiedlicher Forschungspraktiken, welche die Grenzen zwischen Wissenschaft und Gesellschaft überwinden und in partizipativer Weise nach Ansätzen zur konstruktiven Thematisierung und Behebung drängender gesellschaftlicher Problemlagen suchen. Eine gewisse Schnittmenge beider Strömungen ist vorhanden, wenngleich zahlreiche Aspekte noch nicht belichtet wurden (vgl. Räuchle \& Schmiz 2019: 7 f.). Urban Transition Labs sind Experimentier- und Reflektionsorte für alternative Imaginationen und Praktiken. Die Bandbreite von Laborformaten bewegt sich dabei von empowernden Settings bis hin zu solchen, die auf die „Produktion von [...] evidenzbasierten Lösungen“" (Räuchle \& Schmiz 2019: 8) abzielen. Während Labore damit eher infrastrukturelle Rahmungen für transformative Prozesse unterschiedlicher gesellschaftspolitischer Vorzeichen sein können, umfasst die Aktionsforschung explizit zahlreiche und teils widersprüchliche Facetten von Wissenschaft. Unter diesem Sammelbegriff finden sich Ansätze, die von partizipatorischen Erhebungen, die „Beforschte“ eher als Partnerinnen und Partner der Forschung denn als Datenquelle betrachten, bis hin zu „militanten Untersuchungen" reichen können, in denen politisches Engagement und kritische Wissensgenerierung in einem Prozess zusammenfallen sollen (dazu Halder 2018: $45 \mathrm{ff}$.$) .$

Gemein ist den beiden Ansätzen, dass sie anstreben, in aktuelle Problemlagen zu intervenieren. Hier tritt als eines der drängendsten Probleme für ein progressives Gesellschaftsverständnis zuletzt unausweichlich die zunehmende Wortergreifung von Rassisten und Rassistinnen in den Fokus. So instrumentalisierte etwa im Sommer 2018 ein breites Bündnis der radikalen Rechten in Chemnitz den Tod von Daniel H., woraufhin tagelange Ausschreitungen folgen, die anfangs an die Dynamik von Lichtenhagen '92 erinnern. Dominik Intelmann geht in einem lesenswerten Aufsatz auf raumsensible Weise der Frage, weshalb die rechte Massenmobilisierung gerade in der drittgrößten sächsischen Stadt so erfolgreich sein konnte. Unter anderem macht er den Zustand der Zivilgesellschaft als eine Ursache des Problems aus: Ihre Institutionen seien nicht in der Lage gesellschaftlichen Konsens herzustellen, da sie vor Ort zum Teil als „westdeutsch dominiert" und „mit einer eigenen, fremd bleibenden Rationalität betrachtet" werden würden (Intelmann 2019: 192f).

Es dauerte einige Tage, bis sich diese Zivilgesellschaft aufrappelte und die Teilnehmendenzahlen der antifaschistischen und -rassistischen Gegendemonstrationen mit denen der aufgebrachten Rechten mithalten konnte. Eine Woche nach den ersten rassistischen Kundgebungen konnte dann ein Konzert gegen rechts unter dem Motto „Wir sind mehr" organisiert werden, welches 65000 Besucher/innen anzog und dessen zugehöriger Hashtag (\#wirsindmehr) zum meistdiskutierten Hashtag des Jahres avancierte (Spiegel 2018).

Vor dem beschriebenen Hintergrund schien es uns in der Lehrplanung unausweichlich, die rassistischen Ausschreitungen in Chemnitz, die sich entgegenstellenden solidarischen Alternativen und die polarisierte Stimmung in Deutschland in Bezug auf Migration zu behandeln. Dabei unterstützt wurden wir durch ein ad hoc Vernetzungstreffen von Forschenden aus dem Bereich der Geographischen Migrationsforschung, das sich in Osnabrück darauf einigte, das Themenfeld anti-migrantische Mobilisierung verstärkt in der Hochschullehre zu behandeln. In mehreren Seminaren verschiedener Universitäten wurde und wird mittels forschender Lehrmethoden das Thema aufgegriffen und der Verbund tauscht sich regelmäßig über Erfahrungen und Ergebnisse aus.

\section{Fallbeispiele}

Die beiden hier vorgestellten Seminare arbeiteten im Sommersemester 2019 besonders eng zusammen, vertraten beide den Anspruch, innerhalb des Seminarkontextes in urbane Räume zu intervenieren, und stellten eine enge Lehrkooperation zwischen Studierenden in Chemnitz und Berlin her, weshalb sie im Folgenden genauer dargestellt sein sollen.

\subsection{Seminar „Rechtspopulismus in Suburbia“ an der HU Berlin}

Das Seminar an der HU Berlin, das sich an MasterStudierende des Studiengangs „Urbane Geographien“ richtete, hatte zur Vorüberlegung, die vielfältigen Verbindungen zwischen rassistischer Agitation und Themen, vor Ort' zu erarbeiten. Wenn man rassistische und sexistische Positionen als Kern der rechtspopulistischen Deutungsangebote ausmacht, ist es doch noch weitestgehend unklar, welche lokalen Kontexte dafür 
entscheidend sind, dass solche Erzählungen als plausibel und wählbar erscheinen (vgl. Belina 2017: 102). Lokale Konfliktlagen, wie angespannte Wohnungsmärkte, Dieselfahrverbote oder Sicherheitsempfinden in bestimmten öffentlichen Räumen zeigten sich vermehrt für rechte Mobilisierung zugänglich und zugleich während der Konzeptionsphase forschungsseitig unterrepräsentiert (inzwischen liegen hierzu mehr empirische Beobachtungen vor, vgl. etwa Bescherer et al. 2018; Mullis 2019; Üblacker \& Lukas 2019).

In der gemeinsam mit Prof. Dr. Henning Nuissl organisierten Lehrveranstaltung mit 20 Teilnehmenden sollten daher die Formationen lokaler Rechtspopulismen, antimigrantischer Mobilisierung und autoritärer Zuspitzungen in Gemeinden in Berliner Stadtrandlagen untersucht werden. Der räumliche Fokus ergab sich aus wahlgeographischen Beobachtungen und einer internationalen Debatte zum revanchistischen Geist von suburbanen Gebieten (vgl. Niedt 2006; van Gent et al. 2014). Zudem fanden im Verlauf des Seminars Kommunalwahlen in Brandenburg statt, was ein Moment verstärkter Mobilisierung von rechts im Berlin umgebenden Bundesland in Aussicht stellte.

Das Seminar bestand aus drei Formaten: Erstens sollte eine umfassende Auseinandersetzung mit Schlüsseltexten zum Thema das Feld erschließen, Kritik anregen und Fragen aufwerfen. Zweitens waren die Studierenden angehalten, Forschungsdesigns und kleinere (und ggf. transformativ angelegte) Fallstudien zu erstellen und durchzuführen. Drittens wurden in den letzten Sitzungen die Ergebnisse ausgewertet und untereinander sowie mit anderen Fallstudien verglichen. In diesem Format fanden auch zwei Videogruppenkonferenzen mit den Seminarteilnehmenden der TU Chemnitz statt.

Um die Studierenden zu bemächtigen, die Seminargestaltung in die eigene Hand zu nehmen, wurden verschiedene organisatorische Arbeitsgruppen gegründet, von der sich eine um die Ergebnissicherung auf dem gemeinsamen Blog bemühte. Dieser wurde als Studienprojekt an der TU Chemnitz eingerichtet, um die Projektarbeiten der dortigen Seminare zu diesem Themenfeld vorzustellen, wurde aber auch als Austauschplattform für das gemeinsame Lehrforschungsprojekt genutzt (Blog Umkämpfte Geographien o. J.).

In den Lektüreanteilen des Seminars konnte allerdings, trotz vielfältiger didaktischer Methoden, das machtvolle Verhältnis von Dozierenden und Studierenden kaum aufgehoben werden - zu stark ist das Gefälle zwischen demjenigen, der alle Seminartexte aus der Fülle akademischer Literatur ausgewählt hatte und selbst in diesem Feld forscht und Teilnehmerinnen und Teilnehmern, die die Texte z.T. nur auf der Fahrt von Lohnarbeit zum Seminar lesen konnten. Hingegen setzten die Methodenformate umso stärker auf partizipative Wissensproduktionen: Mittels Kurzinputs stellten Studierende ihre Ideen zum Umgang mit dem Phänomen, ihre Forschungsfragen und methodischen Erfahrungen im Plenum vor. Kommentare aus der Gruppe sollten daraufhin einen Lernprozess für alle Beteiligten in Gang setzen.

In den vierstündigen Sitzungen wurde immer wieder die Rolle der Forschenden im Feld und die partizipativen Potentiale von (Aktions-)Forschung diskutiert. Das Gros der neun Forschungsprojekte zog sich allerdings auf eine eher kritisch-beobachtende Position ihres Forschungszugangs zurück - zumeist aus dem Grund, dass sich die partizipativen Ansätze der Aktionsforschung nicht ohne weiteres auf Untersuchungen von rechten Mobilisierungen übertragen lassen und der zeitlich begrenzte Rahmen eines Seminars nicht den nötigen Platz bietet, der notwendig wäre, um diese Fragen zufriedenstellend zu bearbeiten (siehe Kap. 3.1).

Viele Arbeiten zeichnen sich dennoch durch eine hohe Praxisrelevanz aus. So untersuchte etwa eine Gruppe angehender Lehrer/innen die Ansprache junger Menschen durch die Jugendorganisation der AfD, Junge Alternative. Stärker auf den transformativen Anspruch von Aktionsforschung ging eine andere Gruppe ein, die sich auf zentrale Plätze einer suburbanen Gemeinde mit einer Collage positionierte, die Positionen lokaler Rechtspopulistinnen und Rechtspopulisten wiedergab und Passantinnen und Passanten damit konfrontierte. Dies resultierte unter anderem darin, dass einige Befragte wenigstens kurzzeitig auf die Bedrohung vor Ort für Minderheiten aufmerksam gemacht wurden und Prozesse des „Nicht-WahrhabenWollens" in der idyllischen Kleinstadt offensichtlich wurden. Die Gespräche der 38 dokumentierten Begegnungen konnten zudem transkribiert und ex post analysiert werden. Zwei weitere Projekte widmeten sich demgegenüber der Unterstützung von progressiven Kräften in dem Feld, indem sie gemeinsam mit Akteurinnen und Akteuren vor Ort, die sich gegen rechts einsetzen, die jeweils besonderen Bedingungen der Arbeit und Potenziale in randstädtischer und suburbaner Lage erarbeiteten (vgl. Ammon et al. 2019).

\subsection{Seminar „Migration und Solidarität“ an der TU Chemnitz}

Das Lehrforschungsseminar „\#unteilbar, \#wirsindmehr - auch im Wahljahr 2019?" ging Fragen an der Schnittstelle von Migration und Solidarität nach. Neben den o.g. Kundgebungen und dem Konzert, das unter dem Hashtag \#wirsindmehr in Social Media sichtbar wurde, führten die rassistischen körperlichen Übergriffe, Parolen und der Angriff auf Restaurants beim Chemnitzer Stadtfest 2018 auch zur Gründung 
neuer Initiativen sowie zahlreichen Statements durch Institutionen und Vereine. Die rassistischen und rechtsmotivierten Angriffe (u. a. Körperverletzungen, Nötigungen und Bedrohungen) setzten sich dabei auch in der Folgezeit fort, sodass die Beratungsstelle des Vereins RAA Sachsen (2019) im Jahr 2018 eine Vervierfachung in Chemnitz registrierte. Mit Verweis auf existierende Arbeiten über „offene, versteckte und negierte Alltagsrassismen" (Friese et al. 2019: 8; Herv. i. Orig.) ,nach Chemnitz sowie auf gesellschaftsfähige "rassistische Deutungs- und Urteilsmuster" (Castro Varela \& Mecheril 2016: 8), widmete sich das Seminar dem Begriff der "Solidarität" sowie davon ausgehenden gesellschaftlichen Visionen, Allianzen und Praktiken (vgl. u. a. Foroutan 2018; Kron \& Lebuhn 2018; Schilliger 2018; Stjepandic \& Karakayali 2018). Somit wird Kritik an gegenwärtigen Zuständen mit zukunftsgerichteten Perspektiven als sich ergänzende Pfeiler emanzipativer gesellschaftlicher Transformation gesehen (vgl. Brand 2014: 274). Ob und wie solidarisches Handeln in Chemnitzer Bündnissen, Institutionen und digitalen Räumen auch im Frühsommer 2019 fernab medial-öffentlicher Wahrnehmung relevant ist und welche Visionen und Handlungswege für ein solidarisches Miteinander hierbei imaginiert bzw. beschritten werden, waren die zentralen Fragen des Seminars.

Die Vorgehensweise im Seminar folgte der Methode der sog. Urban Transition Labs, die im Bereich der Nachhaltigkeitsforschung entwickelt wurden. Sie können „als hybride, flexible und transdisziplinäre Plattformen, [die] Zeit und Raum zum Lernen, zur Reflektion und zur Entwicklung von alternativen Lösungen für [P] robleme zur Verfügung stellen" (Schäpke et al. 2017: 35) verstanden werden. Diese Lern- und Experimentierräume sind in Bezug auf Größe, Zusammensetzung, Dauer sowie Akteurinnen und Akteure unterschiedlich gestaltet, wie sich auch an den Projektgruppen im Seminar zeigte (s. u.). Im Gegensatz zu kommerziellen Laboren intervenieren Forschende hier in lokale Aushandlungen und orientieren sich dabei an lokalgesellschaftlichen Bedürfnissen. Labore haben dabei das „Doppelziel des Verstehens und der Gestaltung von Wandel“ (Schäpke et al. 2017: 12): Zum einen geben Forschende in diesen Settings Impulse für alternative Lösungen im jeweiligen Kontext und begleiten deren Umsetzung; zum anderen wird das generierte Wissen der wissenschaftlichen Debatte zugeführt. Da die Forschungserfahrung der Stadt- und Migrationsforschung mit Labormethodologien noch recht gering ist (vgl. Räuchle \& Schmiz 2019: 3), war das Seminar selbst als Testplattform und Lernprozess angelegt. Im Seminar wurden zwei Aspekte besonders in den Blick genommen: Erstens konzipierten die Studierenden Experimente mit alternativen solidarischen
Praktiken und Narrativen in konkreten Settings und führten diese durch. Relevant war hierbei nicht, dass die Experimente „erfolgreich“ sind. Vielmehr standen die Reflexion der Experimente, in die Akteurinnen und Akteure aus Zivilgesellschaft, Wissenschaft und Schule involviert waren, und der Verlauf der Urban Transition Labs im Vordergrund.

Zum anderen orientierten sich die Arbeitsgruppen an den drei folgenden Wissensarten: dem Systemwissen, welches den Status quo kritisch reflektiert; dem Orientierungswissen, welches nach Visionen fragt; und dem Transformationswissen, welches die Mittel und Wege zum Erreichen der Visionen hervorbringt (vgl. Schäpke et al. 2017: 37). Diese Aufteilung trägt dazu bei, die kritische Analyse in Zusammenhang mit solidarischen Visionen und machbaren Handlungspfaden zu denken. Die Anwendung der Labormethodologie wurde durch Monatsziele und Feedbackrunden in den Seminarsitzungen vorstrukturiert und die Studierenden erhielten Tools zur Projektorganisation für die hauptsächlich selbstorganisierten Arbeitsphasen. In diesen wurde die Entscheidungs- und Definitionsmacht hinsichtlich relevanter Themen und Vorgehensweisen den Studierenden übertragen. Im Seminar bildeten sich insgesamt fünf Projektgruppen zu folgenden Themen: Social Media, Ausländerbehörde, Universität, Schule, und Zivilgesellschaft. Die letztgenannte Gruppe wird im Folgenden vorgestellt, um einen Einblick in die Umsetzung der Urban Transition Labs zu geben.

Die Projektgruppe Zivilgesellschaft beschäftigte sich mit der Frage, wie die Sichtbarkeit und das Engagement für eine solidarische Stadtgesellschaft erhöht werden kann und welche Unterstützung hierbei notwendig ist. Eine Befragung zu Semesterbeginn auf einer Demonstration in Chemnitz setzte dabei den Ausgangspunkt für weitere Überlegungen. Mithilfe von Postkarten und der Aufforderung zum Ausfüllen: „Solidarität in Chemnitz. Frag was oder sag was“, trat die Projektgruppe mit Teilnehmenden der Demonstration in Interaktion. Anschließend werteten sie die insgesamt 70 erhobenen Antworten aus und stellten die Ergebnisse lokalen Initiativen zur Diskussion. Anhand dieser schnellen und niederschwelligen Sondierung im Feld konnten die Studierenden eine fehlende Vernetzung, mangelnde öffentliche Aufmerksamkeit sowie den Bedarf nach einer Überblickssammlung zu lokalen Engagementmöglichkeiten feststellen. In den folgenden Sitzungen setzte sich die Gruppe mit ansprechenden und praxisfähigen Formaten auseinander und konzipierte einen sogenannten „Solidar-O-Mat“ zu ortsansässigen Initiativen und Vereinen aus den Bereichen Kultur, Antidiskriminierung und Demokratieförderung. Auf Basis von sechs Interviews zu Aspekten wie Aktionsfelder, Verständnis von Solidari- 


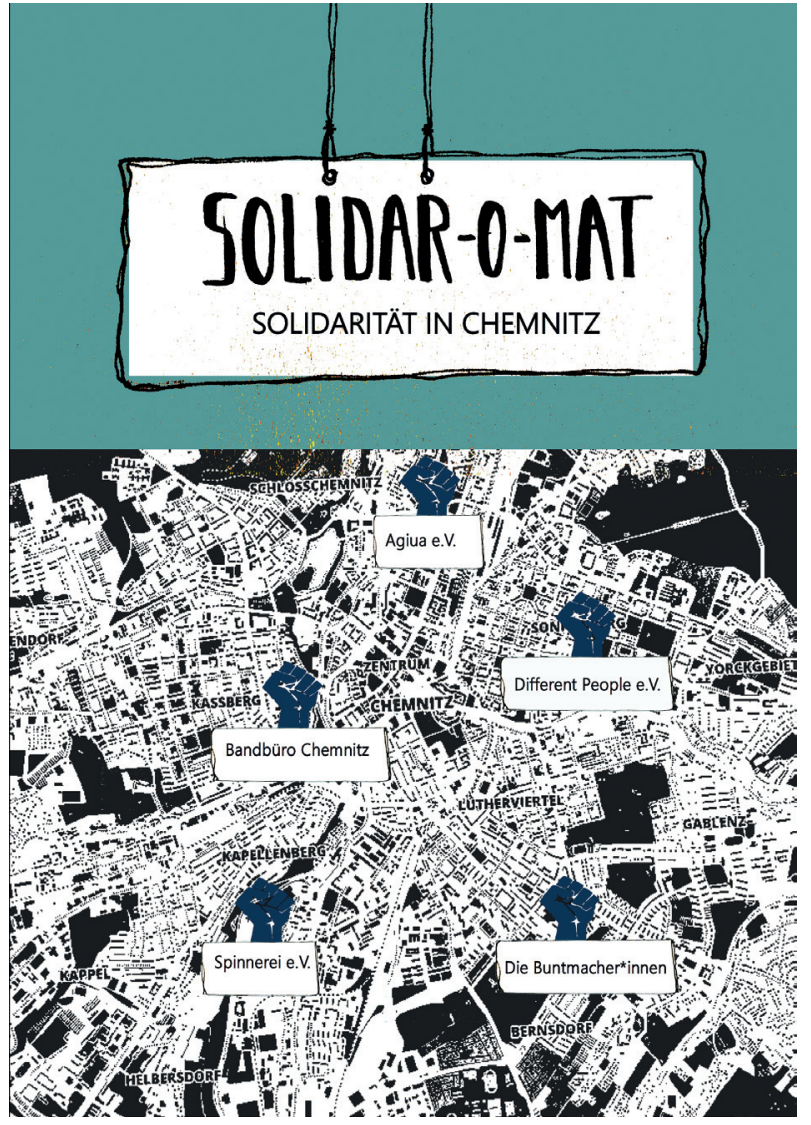

Abb. 1: „Solidar-O-Mat“, Titelbild der Broschüre. Der Titel knüpft an den Wahl-O-Mat der Bundeszentrale für politische Bildung an. Quelle: Darstellung der Projektgruppe.

tät, Zukunftsvisionen und Engagementmöglichkeiten designte die Projektgruppe eine analoge Broschüre (siehe Abb. 1). Dieses Zwischenergebnis dient der Präsentation der Idee des Solidar-O-Mats, der in der Folgezeit des Seminars wachsen, bekanntgemacht und digitalisiert werden soll.

\section{Diskussion}

\subsection{Positionierungen als Studierende, Forschende, Engagierte}

Während sich kritisch-emanzipatorische Ansätze zunächst harscher Kritik gegenübersahen, da positivistische Grenzen zwischen Forschungssubjekt und -objekt, Forschenden und Beforschten infrage gestellt wurden, erfuhren solche Ansätze in kritischen Gesellschaftswissenschaften vermehrt Anerkennung (vgl. Halder 2018: 37 ff.). Ihre Anwendung in Kontexten einer polarisierten Gemengelage, wie sie spätestens nach den Ausschreitungen in Chemnitz konstatiert werden können, sowie in Forschungen über potenzielle Zukunftsszenarien ist jedoch herausfordernd.
Auf der einen Seite konnte im Berliner Seminar beobachtet werden, wie diese Ansprüche scheitern, wenn in den beforschten Konstellationen rassistische und menschenverachtende Positionen dominant sind. In der Konzeptionsphase einiger Studienprojekte wurde deutlich, wie schwer der partizipatorische Gedanke von Aktionsforschung, kollaborativ mit „den Beforschten" neues Wissen zu produzieren, sich in die Praxis umsetzen lässt. Die Herausforderung, ,mit Rechten zu reden' und zu Rechten zu forschen, wird im sub/urban-Themenheft "Stadt von rechts" ausführlich diskutiert. Robert Feustel weist dabei auf nahezu unüberwindbare Forschungsbarrieren zwischen an emanzipatorischen Werten ausgerichteten Forschenden und der neuen Rechten und ihren Anhängerinnen und Anhängern hin. Einerseits ergäben sich diese aufgrund kontrafaktischer Tiefenstruktur neurechten Denkens und Argumentierens, die sich gegenüber faktenbasierter Analyse nur wenig zugänglich zeigen. Anderseits erschwere die Positionalität von Universitätsangehörigen und ihre Bedeutungszuschreibung in neurechten Diskursen eine Auseinandersetzung, die nicht von gegenseitigen Vorverurteilungen geprägt ist, massiv (vgl. Feustel 2019). Auch wenn es hilfreiche Hinweise gibt, unter welchen Prämissen kritische Forschung vor Ort möglich ist (vgl. etwa Quinn 2019), entschied sich das Gros der Berliner Studierenden doch für klassische Forschungszugänge, die den obigen Fallstricken aus dem Weg gehen. Während zu Beginn des Seminars der Wille vor Ort zu forschen noch bei allen Gruppen sehr präsent war, nahm diese Bereitschaft mit zunehmender Auseinandersetzung mit dem Thema bei einigen Gruppen $\mathrm{ab}$, sodass schlussendlich einige beobachtende Analysen überwogen, die allerdings von hoher Qualität und tiefgehender Auseinandersetzung mit dem Thema geprägt waren. Eine Arbeit konnte etwa mittels einer Cluster-Analyse die Repräsentativität von AfD-Kandidatinnen und -Kandidaten in Hinblick auf ihren Wahlerfolg befragen (Goerlich \& Kormeyer 2019).

Auf der anderen Seite wurde im Seminar in Chemnitz deutlich, wie die Zusammenarbeit mit außeruniversitären Akteurinnen und Akteuren mitunter von dem im Hochschulkontext erwarteten Output abhängig gemacht wurde. Die Studierenden wurden durch den Rahmen des Urban Transition Lab dazu ermutigt, Forschung als einen ergebnisoffenen Prozess zu verstehen, in welchem die Kritikerin bzw. der Kritiker die Position des distanzierten Beobachtens und Analysierens verlässt und übergeht zu einem kritischen Forschen, das „einen Rahmen [zur Reflektion und Schaffung von Alternativen] schafft" (Terkessidis 2015: 297). Die hierdurch möglicherweise evozierte Rollendiffusion im Forschungsprozess (vgl. Räuchle \& Schmiz 2019: 9) äußerte sich bei den Projektgrup- 
pen vereinzelt in dem Gefühl, zur Schaffung von vorzeigbaren Outputs und tatsächlichen Veränderungen aufgefordert zu sein. Dadurch wurden gescheiterte Experimente als Misserfolge präsentiert statt die als Lernanlass um die Wirkweisen von Veränderungen zu betrachten. Gewissheit hinsichtlich des Prozessverlaufs erlangten Gruppen auch dadurch, dass sie das Labor tendenziell eher mit der eigenen Projektgruppe und dem Seminar gleichsetzten und sich nicht in einen gänzlich kollaborativen Aushandlungsprozess mit Praxisakteurinnen und Praxisakteuren begaben. So behielten sie die Definitionsmacht über die Prozessschritte innerhalb der Projektgruppe und traten vor allem für Interviews und die Besprechung der Untersuchungsergebnisse mit externen Praxisakteurinnen und -akteuren in Kontakt. Das Feedback der Studierenden weist darauf hin, dass der Dozent die Studierenden noch stärker auf die Relevanz des ergebnisoffenen Seminarcharakters hätte aufmerksam machen können.

Aus dem eigensinnigen Umgang der Studierenden mit den Forschungsmethodologien lässt sich schließen, dass keine Anwendung der transformativen Ansätze in Reinform im Seminarkontext zu erwarten ist. Nimmt man den Anspruch eines möglichst selbstbestimmten (forschenden) Lernens und Lehrens ernst (vgl. Heyman 2001), gilt es, die eigenen Intentionen als Dozent/in hinten anzustellen und die Studierenden ihre Forschungszugänge selbst wählen zu lassen.

\subsection{Methodologischer Kontext der Interventionen}

Auch wenn die Studierenden, die jeweils von uns Dozenten vermittelten Forschungsprogramme unterschiedlich umgesetzt haben, verweisen die Seminarverläufe auf ähnliche Konturierungen und Ambivalenzen der Forschungsprozesse und -interventionen. So dienten die Konzepte des Urban Transition Lab und der Aktionsforschung jeweils als raum- und zeitstrukturierende Plattformen, welche einen Austausch mit externen Akteurinnen und Akteuren sowie zwischen alltagsweltlichen Interventionen und theoretischen Ansätzen ermöglichen sollten.

Dabei zeigte sich, dass die Studierenden innerhalb der Methodologie des Urban Transition Lab eher die Pfade klassischer Erhebungen verlassen haben. Auch wenn in den Projekten die Gefahr der Reaffirmation gesellschaftlicher Verhältnisse oder der Reduktion von Veränderungen auf das Mögliche bestand, wurden die Studierenden selbst Teil von Interventionen und Veränderungsprozessen, die durch ihre Gruppenarbeit Gestalt annahmen und damit erlebbar und erfahrbar wurden. Dies war in gewisser Weise auch durch den Seminarcharakter hinsichtlich Zeitressourcen und Abhängigkeitsverhältnisse zwischen Studierenden und Dozierenden bedingt.
In den Projektgruppen waren die Interventionen durch das Spannungsfeld zwischen Reproduktion und Veränderung gesellschaftspolitischer Verhältnisse geprägt. Indem sich die Gruppen mit bestehenden lokalen Institutionen, Diskursen und Praktiken beschäftigen und durch alternative Denk- und Handlungsweisen intervenierten, arbeiteten sie einerseits anwendungsbezogen, da sie entlang alltäglicher Bedarfe in den jeweiligen Kontexten realistisch praktizierbare Lösungen konzipierten. Andererseits war die Herangehensweise auch kritisch, wenn sie die vorgefundenen sozialen Verhältnisse hinterfragten und durch die Interventionen auf deren Veränderbarkeit verwiesen (vgl. Glasze 2012: 162). In den Seminaren standen dabei die erwirkten Veränderungen weniger im Vordergrund als der Prozess der theoretischen und Empirie-basierten Reflexion sozialer Verhältnisse sowie der Imagination von und dem Experimentieren mit Alternativen.

Durch die Orientierung an der Laborforschung konnten sowohl Forschungsfragen als auch Auswertungen gewissermaßen vorstrukturiert werden. Beispielsweise verwendeten alle Gruppen die Aufteilung der o.g. transformativen Wissensarten. Stand diese Kontextualisierung nicht zu Beginn des Seminars fest, sondern stellte sie sich erst in dessen Verlauf als hilfreich heraus, so half sie gegen Ende beim Vergleich und der Einordnung der Ergebnisse. Gleichwohl weist diese Vorgehensweise formalistische Züge auf und birgt die Gefahr, gesellschaftspolitische Machtverhältnisse in ein zu enges Denkkorsett zu pressen. Ansätze der Aktionsforschung dagegen rücken stärker die gesellschaftspolitischen Dimensionen von Interventionen in den Vordergrund. So ist „transformative Gültigkeit[, die] beschreibt, inwieweit die Forschung als ein Beitrag zum gesellschaftlichen Wandel verstanden werden kann", als eines der zentralen Gütekriterien von Aktionsforschung zu verstehen (Halder 2018: 76). Jedoch ließen sich auf die recht abstrakten Ideen der Aktionsforschung, die ohne festes konzeptionelles Gerüst auskommen, nur wenige der Berliner Studierenden ein.

\subsection{Positionierungen im institutionellen Setting der Hochschullehre}

Die Umsetzung der beiden Methodologien im universitären Lehrbetrieb war nicht zuletzt möglich, da wir auch Veränderungen am Seminarsetting an sich vornahmen. Erstens waren die Studierenden in Bezug auf die Einteilung ihrer Ressourcen vor die Herausforderung gestellt, neben der Lektüre einschlägiger Fachtexte auch empirische Erhebungen, Interventionen und Experimente zu designen und umzusetzen, sowie sich dafür in kurzer Zeit neue Fähigkeiten anzueignen (bspw. Moderation von Workshops) und 
mitunter Kontakte zu Akteurinnen und Akteuren im Feld aufzubauen. Die Verknüpfung dieser vielfältigen Anforderungen folgte dabei dem Ziel, „einen pragmatischen Rahmen für Veränderung" (Terkessidis 2015: 12) innerhalb komplexer Forschungsprozesse zu gesellschaftspolitischen Aushandlungen zu schaffen. Auf Seiten der Dozierenden stellte sich insbesondere die Frage nach deren Rolle, die sie mehr als Begleiter der Gruppenprozesse und Moderatoren für den Wissenszuwachs der Studierenden verstanden. Die Rolle als Fachexperten nahmen sie eher auf Nachfrage wahr, statt diese aktiv auszuleben. Dieses Rollenverständnis brachte eine Auseinandersetzung mit didaktischen Mitteln der Prozessgestaltung sowie eine Distanzierung von einem (be-)lehrenden DozentenDuktus mit sich.

Auch wenn konventionelle Hierarchie-Verhältnisse zwischen Studierenden und Dozierenden an manchen Stellen zumindest in Frage gestellt werden konnten, zeigte sich, zweitens, immer wieder der starke Einfluss der Positionalitäten der Beteiligten auf die Lehrsituation. Rich Heyman (2001) fordert auch daher ein besonderes Augenmerk darauf zu legen, die formalen Aspekte der Begegnung im Seminar dahingehend zu transformieren. Demnach sollen diese mehr als Orte der lebendigen öffentlichen Debatte fungieren, denn dem Zweck dienen, Studierenden objektiviertes Wissen einzutrichtern. In unseren Seminarkontexten, die von sich überlagernden und teils widersprüchlichen Machtpositionen gekennzeichnet waren, spielten diese Überlegungen eine ganz maßgebliche Rolle. Mit Blick auf den Beutelsbacher Konsens hielten wir es dabei mit Gill Valentine: ,as teachers we have a responsibility to give students the skills to navigate their own ethical maps" (Valentine 2005: 486). Dieser Ansatz war fortlaufend von herausfordernden Situationen begleitet.

Allein durch unsere klaren Positionierungen gegenüber dem Aufstieg rechtsradikaler Kräfte bzw. der Betonung solidarischer Bewegungen in den Seminarbeschreibungen war die Stoßrichtung vorgegeben. Dabei wurden verschiedene Positionen untereinander und zu den Dozierenden ständig neuverhandelt - insbesondere bei den polarisierenden Seminarthemen. In beiden Seminaren konnten mehr oder weniger starke Reibungsprozesse innerhalb der Gruppen beobachtet werden, von denen wir annehmen, dass sie die Auseinandersetzung mit der übergeordneten gesellschaftspolitischen Thematik vertieft. Diese Beobachtungen decken sich mit der Einschätzung von John Paul Catungal (2019: 46): „One important way that power/ knowledge manifests in and shapes the classrooms is through the negotiation of the relational identities of subjects-including teachers and learners-in these educational spaces."

\section{$4 \quad$ Ausblick und Appell}

In den beiden vorgestellten Seminaren war der Lehrkontext ein Ort für eigensinnige studentische Aneignungen von Forschungsmethodologien, für die Erprobung von Interventionen mit ambivalenten Forschungsprogrammen und zur (Neu-)Positionierung von Studierenden und Lehrenden. Nicht unterschlagen wollen wir dabei, dass der Seminarraum auch ein Ort ungleicher Arbeitsverhältnisse ist (vgl. Catungal 2019: 47). Alle Beteiligten müssen die Ressourcen, die sie für eine Lehrveranstaltung aufwenden, hinsichtlich ihres Verhältnisses zur Lohnarbeit bewerten: Studierende danach, wie verwertbar die angeeigneten Skills auf dem Arbeitsmarkt sind und das akademische Personal hinsichtlich des Raumes, den die Lehre gegenüber anderen Aufgaben einnimmt. Dies schränkt die Möglichkeiten freier Seminargestaltung von beiden Seiten ein - und wir als Lehrende sind uns bewusst, dass derartig aufwendige Veranstaltungen oft mit unbezahlter Mehrarbeit einhergehen.

Doch auch wegen dieser und den oben ausgeführten Widrigkeiten sind wir davon überzeugt, dass Lehrformate, die den klassischen Seminarraum verlassen auch, vielleicht sogar ganz besonders unter Bedingungen polarisierter gesellschaftlicher Verhältnisse gewinnbringend für Erkenntnisprozesse von Studierenden sein können. Der Appell, den solche Formate an die Studierenden richten, sich ihrer Position als Universitätsangehörige bewusst zu werden und ihre Rolle als Forschende zu reflektieren, wurde in den Kontexten, in denen sich die Seminare bewegten, forciert. Die gesellschaftliche Involviertheit, in die sich die Teilnehmer/innen zwangsläufig zu einem gewissen Grad begaben, führte sie zudem auch an Nuancen verschiedener Machtverhältnisse heran, die durch die reine Lektüre von Fachliteratur nicht erschließbar ist. Dies geht auch aus dem positiven Feedback der Studierenden hervor, von denen viele einen großen Wissenszuwachs im Umgang mit herausfordernden und widersprüchlichen Forschungsprozessen angeben.

Demnach hat die institutionalisierte kritische Geographie gerade dort ihren größten Mehrwert, wo sie institutionelle Praktiken hinterfragt und in gesellschaftspolitische Themen interveniert. Die jahrzehntelangen Erfahrungen mit Instrumenten eingreifender Forschungspraktiken sollten nicht über Bord geworfen werden, sobald regressive Akteure und Akteurinnen sowie Positionen in den Fokus der Forschung rücken: Es gilt, sich nicht auf objektivistische Positionen zurückzuziehen, gegen die Teile der kritischen Geographie sich lange behaupten mussten. Folglich verstehen die Autoren diesen Text nicht nur als Seminaranalyse, sondern vielmehr als Intervention in die 
Praxis der Hochschullehre an sich. So rufen wir zum Schluss mit Desbiens und Smith (1999: 382) zu einer kritischen Lehrpraxis auf, die gesellschaftliche Aushandlungsprozesse, Widersprüche und Konflikte thematisiert und erlebbar macht und dabei gleichzeitig die Grenzen des Seminarraums überschreitet: „We are critical because we refuse the self-imposed isolation of much academic research, believing that social science belongs to the people and not the increasingly corporate universities."

\section{Acknowledgement}

Wir danken für die Unterstützung durch die Deutsche Forschungsgemeinschaft (DFG) und den OpenAccess-Publikationsfonds der Humboldt-Universität zu Berlin.

\section{Literatur}

Ammon, I., D. Barahona, L. Hagenlocher \& N. Oltersdorf (2019): Mobilisierung gegen rechts im suburbanen Raum. https://blog.hrz.tu-chemnitz.de/umkaempftegeochemnitz/andere-standorte/mobilisierung-gegen-rechtsim-suburbanen-raum/ (28.11.2019)

Belina, B. (2017): Zur Geographie der Abstiegsgesellschaft. PROKLA. Zeitschrift für kritische Sozialwissenschaft 47(186). S. 97-104.

Bescherer, P., R. Feustel, L. Schelenz \& L. Sievi (2018): Urbaner Populismus? Das Gefahrenpotenzial der Stadtentwicklung. Populismus und Demokratie in der Stadt. PODESTA-Working Paper 1. http://podesta-projekt. de/wp-content/uploads/2018/08/Urbaner-PopulismusWorkingPaper-1-Podesta-August-2018-web.pdf(27.11.2019)

Blog Umkämpfte Geographien (o. J.): Startseite. https:// blog.hrz.tu-chemnitz.de/umkaempftegeo-chemnitz/ (27.11.2019)

Brand, U. (2014): Transition und Transformation: Sozialökologische Perspektiven. In: Brie, M. (Hg.): Futuring. Perspektiven der Transformation im Kapitalismus über ihn hinaus. Westfälisches Dampfboot, Münster. S. 242-280.

Castree, N. (2000): Professionalisation, Activism, and the University: Whither 'Critical Geography'? In: Environment and Planning A: Economy and Space 32(6). S. 955-970.

Castro Varela, M. do M. \& P. Mecheril (Hrsg.) (2016): Die Dämonisierung der Anderen. Einleitende Bemerkungen. In: Die Dämonisierung der Anderen: Rassismuskritik der Gegenwart. transcript, Bielefeld. S. 7-19.

Catungal, J. P. (2019): Classroom. In: Jazeel T., A. Kent, K. McKittrick, N. Theodore, S. Chari, P. Chatterton, V. Gidwani, N. Heynen, W. Larner, J. Peck, J. Pickerill, M. Wernder \& M. W. Wright (Hrsg.): Keywords in Radical Geography: Antipode at 50. John Wiley \& Sons, Hoboken. S. 45-49.
Desbiens, C. \& N. Smith (1999): The International Critical Geography Croup: Forbidden Optimism? In: Environment and Planning D: Society and Space 17(4). S.379-382.

Feustel, R. (2019): Substanz und Supplement. Mit Rechten reden, zu Rechten forschen? Eine Einladung zum Widerspruch. In: sub \urban. zeitschrift für kritische stadtforschung 7(1/2). S. 137-146.

Foroutan, N. (2018): Die postmigrantische Perspektive: Aushandlungsprozesse in pluralen Gesellschaften. In: Hill, M. \& E. Yıldız (Hrsg.): Postmigrantische Visionen. Erfahrungen - Ideen - Reflexionen. transcript, Bielefeld. S. 15-27.

Friese, H., M. Nolden \& M. Schreiter (2019): Rassismus im Alltag: Theoretische und empirische Perspektiven nach Chemnitz. transcript, Bielefeld.

Glasze, G. (2012): Eine politische Konzeption von Räumen. In: Dzudzek, I., C. Kunze \& J. Wullweber (Hrsg.): Diskurs und Hegemonie. Gesellschaftskritische Perspektiven. transcript, Bielefeld. S. 151-172.

Goerlich, M. \& T. Kormeyer (2019): Repräsentativität Der Schlüssel zum Erfolg der Brandenburger AfD bei den Wahlen 2019? https://blog.hrz.tu-chemnitz.de/umkaempftegeo-chemnitz/andere-standorte/repraesentativitaet-als-schluessel-zum-erfolg-der-afd/ (28.11.2019)

Halder, S. (2018): Gemeinsam die Hände dreckig machen: Aktionsforschungen im aktivistischen Kontext urbaner Gärten und kollektiver Kartierungen (PhD Thesis). transcript, Bielefeld.

Hay, I. (2001): Engaging Lessons: Classrooms as Sites of Engagement in Activist Critical Geography. In: International Research in Geographical and Environmental Education 10(2). S. 168-173.

Heyman, R. (2001): Why Advocacy Isn't Enough: Realising the Radical Possibilities of the Classroom. In: International Research in Geographical and Environmental Education 10(2). S. 174-178.

Intelmann, D. (2019): Sieben Thesen zur urbanen Krise von Chemnitz: Bemerkungen zu den Ereignissen seit dem 26. August 2018. In: sublurban. zeitschrift für kritische stadtforschung 7(1/2). S. 189-202.

Kron, S. \& H. Lebuhn (2018): Solidarische Städte: Globale Soziale Rechte und das Recht auf Mobilität. www.rosalux. $\mathrm{de} /$ publikation/id/39274/solidarische-staedte-globalesoziale-rechte-und-das-recht-auf-mobilitaet/ (02.09.19)

Mullis, D. (2019): Urban conditions for the rise of the far right in the global city of Frankfurt: From austerity urbanism, post-democracy and gentrification to regressive collectivity. In: Urban Studies.

Niedt, C. (2006): Gentrification and the Grassroots: Popular Support in the Revanchist Suburb. In: Journal of Urban Affairs 28(2). S. 99-120.

Quinn, R. A. (2019): Mit Rechten reden, zu Rechten forschen? Ein Widerspruch auf Einladung. sub \urban. zeitschrift für kritische stadtforschung 7(1/2). S. 147-152.

RAASachsene.V.(2019): PressemitteilungzurStatistik2018. https://www.raa-sachsen.de/support/pressemeldungen/ pressemitteilung-zur-statistik-2018-3834 (15.11.2019) 
Räuchle, C. \& A. Schmiz (2019): Reallabore in Ankunftsquartieren. IMIS Working Paper 03, Institut für Migrationsforschung und Interkulturelle Studien (IMIS) der Universität Osnabrück. IMIS, Osnabrück. https://www. imis.uni-osnabrueck.de/fileadmin/4_Publikationen/ PDFs/IMIS_WP03_Reallabore_RaeuchleSchmiz.pdf (15.11.2019)

Reinmann, G. (2016): Gestaltung akademischer Lehre: semantische Klärungen und theoretische Impulse zwischen Problem- und Forschungsorientierung. In: Zeitschrift für Hochschulentwicklung 11(5). S. 225-244.

Schäpke, N., F. Stelzer, M. Bergmann, M. Singer-Brodowski, M. Wanner, G. Caniglia \& D. J. Lang (2017): Reallabore im Kontext transformativer Forschung: Ansatzpunkte zur Konzeption und Einbettung in den internationalen Forschungsstand. In: IETSR Discussion Papers in Transdisciplinary Sustainability Research 1.

Schilliger, S. (2018): Urban Citizenship. Teilhabe für alle, da, wo wir leben. In: Aigner, H. \& S. Kumnig (Hrsg.): Stadt für alle! Analysen und Aneignungen. Mandelbaum, Wien. S. 14-35.
Spiegel (05.12.2018): \#WirSindMehr war der populärste Debatten-Hashtag. https:/www.spiegel.de/netzwelt/ web/twitter-das-sind-die-erfolgreichsten-debatten-hashtags-2018-a-1242047.html (05.02.2020)

Stjepandic, K. \& S. Karakayali (2018): Solidarität in postmigrantischen Allianzen: Die Suche nach dem Common Ground jenseits individueller Erfahrungskontexte. In: Foroutan, N., J. Karakayali \& R. Spielhaus (Hrsg.): Postmigrantische Perspektiven. Ordnungssysteme, Repräsentationen, Kritik. Campus, Frankfurt am Main. S. 237-252. Terkessidis, M. (2015): Kollaboration. Suhrkamp, Berlin.

Üblacker, J. \& T. Lukas (2019): Keine Angst, es ist nur Gentrification? In: sub \ urban. zeitschrift für kritische stadtforschung 7(1/2). S. 93-114.

Valentine, G. (2005): Geography and ethics: moral geographies? Ethical commitment in research and teaching. In: Progress in Human Geography 29(4). S. 483-487.

van Gent, W. P. C., E. F. Jansen \& J. H. F. Smits (2014): Right-wing Radical Populism in City and Suburbs: An Electoral Geography of the Partij Voor de Vrijheid in the Netherlands. In: Urban Studies 51(9). S. 1775-1794. 